\title{
ESQUEMAS DE RAZONAMIENTO Y DE ACCIÓN DE ESTUDIANTES DE ESO EN LA INTERPRETACIÓN DE LOS CAMBIOS PRODUCIDOS EN UN SISTEMA MATERIAL
}

\author{
DOMÍNGGEZ CASTIÑEIRAS, JOSÉ M. ${ }^{1}$, DE PRO BUENO, ANTONIO², \\ GARCÍA-RODEJA FERNÁNDEZ, EUGENIO ${ }^{1}$ \\ ${ }^{1}$ Departamento de Didáctica das Ciencias Experimentais. Facultad de Ciencias da Educación. USC. \\ Av. Xoan XXIII, s/n. 15704 Santiago de Compostela \\ ddacabdz@usc.es \\ ${ }^{2}$ Departamento de Didáctica de las Ciencias Experimentales. Facultad de Educación. \\ Campus Espinardo. UM. 30100 Murcia \\ nono@fcu.um.es
}

\begin{abstract}
Resumen. A partir de la teoría de los esquemas, se ha diseñado una nueva estrategia de evaluación -esquemas de razonamiento y esquemas de acción- para valorar el aprendizaje del conocimiento conceptual y procedimental. Tratamos de identificar los posibles esquemas (dilatación y relación entre variables) que usan algunos estudiantes (12-13 años) en la interpretación de hechos relacionados con su vida cotidiana. En concreto, nos hemos centrado en qué ocurre en un sistema material cuando recibe energía transferida mediante un mechero. Por último, establecemos ciertas implicaciones para la enseñanza de estos contenidos en la educación secundaria.

Palabras clave. Esquemas de razonamiento, esquemas de acción, aprendizaje científico, cambios en los sistemas materiales, educación secundaria.

Summary. Starting from the schema theory, an new evaluation strategy has been developed -reasoning schema and action schema- to assess the learning of conceptual and procedural knowledge. We attempt to identify the possible schema (dilation, relation among variables) which some students (12-13 years old) use in their interpretation of some aspects related to their everyday life. In this particular instance, we have concentrated in what happens in a material system when it receives an energy transference with a burner. Finally, we have established certain implications for the teaching of these contents in compulsory secondary education.

Keywords. Reasoning schema, action schema, scientific learning, changes on material systems, secondary school.
\end{abstract}

\section{INTRODUCCIÓN}

El interés por conocer cómo y por qué se produce el aprendizaje sigue vigente, como se ha puesto de manifiesto en la monografía Research In Science Education in Europe (Bandiera et al., 1999) y en las principales revistas del área. En dicho contexto, este trabajo forma parte de una investigación mucho más amplia (Domínguez, 2000) en la que hemos estudiado qué incidencia tiene una determinada propuesta de enseñanza (Domínguez et al., 2001b) en el aprendizaje de unos alumnos y alumnas de 12-13 años, cómo y por qué se producen estos cambios en los conocimientos, y qué queda de lo aprendido al cabo de un cierto tiempo. En un trabajo de estas características y desde la perspecti- va de la instrucción nos interesa, además de averiguar qué sabe el alumnado sobre un contenido concreto, cómo está articulado este conocimiento, cómo lo utiliza y cómo y por qué evoluciona cuando los estudiantes se enfrentan a la interpretación y explicación de hechos y fenómenos físicos.

A este respecto, las aportaciones de las teorías de las concepciones alternativas y del cambio conceptual (Posner et al., 1982; Nussbaum, 1989) no han sido suficientes para comprender cómo los estudiantes construyen sus representaciones mentales, por lo que se han incorporado otras apor- 
taciones de la ciencia cognitiva a la investigación del proceso de construcción de conocimientos de las ciencias.

Desde dicha perspectiva se asume que un modelo mental (Moreira, 1997; Greca y Moreira, 1998; Duit y Glynn, 1996) es una representación implícita del conocimiento de un no-experto (normalmente de un alumno), que suele ser incompleta, imprecisa e incoherente respecto del conocimiento deseable desde la ciencia escolar en los distintos dominios. Son representaciones internas y personales, por lo que, para conocerlos, habrá que hacerlos explícitos. A estos modelos explícitos los hemos denominado esquemas de conocimiento y para elaborarlos hemos tenido que identificarlos y poner de manifiesto los conjuntos de proposiciones que los constituyen, con el consiguiente «ruido» que se produce durante el proceso de elaboración.

Para llevar adelante dicho proceso, hemos acudido a lo que denominamos esquemas de conocimiento: esquemas de razonamiento y esquemas de acción (Domínguez, 2000), que nos han permitido elaborar los esquemas referenciales o representaciones externas, creadas por los investigadores. Son representaciones explícitas, precisas, más completas y estables, consistentes con el conocimiento y compartidas en el ámbito de la ciencia escolar. Dichos esquemas nos han guiado en la secuenciación de los contenidos, en el diseño de las actividades objeto de aprendizaje y en la evaluación del mismo, puesto que a partir de ellos hemos podido elaborar los esquemas de conocimiento de los estudiantes y estudiar su evolución, desde los iniciales hacia los esquemas referenciales (Nerssesian, 1995; Sutton, 1996; Moreira, 1997; Domínguez, 2000).

Además hemos de señalar que los modelos mentales y los procesos de modelización, así como los esquemas de conocimiento, dependen del contexto y del contenido. Esto complica aún más su identificación, ya que problemas, actividades o pruebas diferentes pueden dar lugar a diversos modelos y esquemas. Hemos tratado de resolver este problema mediante lo que hemos denominado dimensionar el conocimiento.

\section{LOS ESQUEMAS DE RAZONAMIENTO Y DE ACCION PARA DIMENSIONAR EL CONOCI- MIENTO}

El conocimiento humano es mucho más rico y complejo que cualquier concepto científico, por lo que resulta mucho más difícil su investigación. Si queremos acercarnos al conocimiento del mismo, necesitamos -entre otras cosas- explorar el razonamiento presente en el discurso del alumnado, así como las estrategias de acción implicadas en el mismo.

Para abordar dicho estudio nos han sido útiles las aportaciones de la teoría de los esquemas, desarrolladas en el marco de la ciencia cognitiva. Esta teoría permite caracterizar la forma en la que se estructura el discurso de los estudiantes, a partir de su razonamiento y de sus acciones. A este respecto hemos considerado las definiciones de es- quema de Rumelhart y Ortony (1982), Rumelhart (1989, 1997), la de guión de Schanck y Abelson (1987) y la de marco de Minsky (1986), que nos parecen bastante coincidentes.

Se considera que los esquemas son estructuras de datos para representar los conceptos genéricos almacenados en la memoria. Constituyen estructuras dinámicas de creciente complejidad, como red de interrelaciones, que no se pueden explicar como una simple suma de los elementos que las constituyen. Pueden representar conceptos, situaciones, sucesos, secuencias de sucesos, acciones o secuencias de acciones.

Poseen unos principios fundamentales:

- El conocimiento está estructurado en unidades llamadas esquemas.

- Los esquemas contienen información del conocimiento y de cómo se usa.

- El procesamiento de la información y la construcción del conocimiento dependen de la activación de los esquemas que tiene el sujeto.

- Cada esquema dominante o principal está caracterizado en términos de constituyentes de nivel inferior, los subesquemas y las variables, además de las relaciones que se establecen entre ellos. Cuando alguno de estos elementos aparece en el discurso diremos que ha sido activado.

Desde esta perspectiva, los esquemas de razonamiento y de acción son representaciones del conocimiento que los estudiantes adquieren y construyen con el aprendizaje y que pueden activar para utilizar o procesar una determinada información. La naturaleza de los esquemas determina que puedan usarse tanto de modo declarativo como procedimental, pues permiten la identificación de problemas, la elaboración de estrategias o la selección de destrezas y técnicas. De esta forma el saber qué y el saber cómo constituyen dos usos distintos de la misma representación (Pozo, 1989).

En este contexto, el aprendizaje significativo resultaría de la evolución de los esquemas (implícitos) que tienen los alumnos antes de nuestra intervención en el aula, hacia los esquemas referenciales (explícitos) deseables desde la ciencia escolar. Desde esta perspectiva nos ha interesado profundizar en cómo actúan en el proceso de comprensión, en el establecimiento de inferencias y en el desarrollo de acciones.

No obstante, al hilo de este marco de referencia, se plantean muchos interrogantes: ¿Cómo hacer explícitos los esquemas implícitos que los estudiantes tienen en un momento concreto y en un determinado dominio? ¿Cómo lograr que sean independientes del contexto (problema) utilizado para activarlos? ¿Cómo estudiar la evolución de los mismos a lo largo de la intervención en el aula? ¿Cómo saber los que han construido como resultado del aprendizaje? ¿Cómo estudiar la estabilidad de los esquemas en el tiempo?... 
Para resolver estos interrogantes hemos optado por dimensionar el conocimiento deseable desde la cienca escolar mediante la elaboración de cuatro esquemas de razonamiento (modelo cinético de partículas, incremento de temperatura, dilatación, cambio de estado) y cuatro esquemas de acción (medida de la temperatura, representación gráfica, relación entre variables y diseño experimental).

En este trabajo se realizan aportaciones, para el contraste de la siguiente hipótesis:

Los esquemas iniciales (i) de razonamiento y de acción de los alumnos de la muestra (12-13 años) están alejados de la ciencia escolar y son poco útiles para la interpretación de los cambios producidos en un sistema material cuando intercambia energía por calor con una fuente (mechero).

\section{LA MUESTRA}

Como uno de los objetivos centrales de la investigación era el estudio de la puesta en práctica de una unidad didáctica en una clase de secundaria, no se ha buscado la representatividad sino una situación natural en la enseñanza. Se trabajó con una muestra incidental (el grupo de clase de la profesora que trabaja en nuestro equipo) y poco numerosa, porque pretendíamos hacer un seguimiento de la evolución de cada uno de los esquemas de los alumnos a lo largo del proceso de enseñanza-aprendizaje y una vez transcurrido un cierto tiempo desde nuestra intervención. Estaba constituido por 8 chicos y 13 chicas, con una edad media de 13 años, de los que 5 habían repetido alguno de los cursos anteriores. Sus padres eran trabajadores y habían realizado preferentemente estudios primarios. Uno causó baja por enfermedad.

\section{ESTRATEGIA DE INVESTIGACIÓN}

Hay numerosas investigaciones que han tratado de conocer qué ideas, concepciones y creencias tienen los estudiantes sobre los mencionados cambios del sistema. En un trabajo anterior (Domínguez et al., 2001a) ya nos detuvimos a analizar algunas de ellas, que hemos sintetizado en el cuadro I.

Estas aportaciones constituyen un punto de partida importante en nuestra investigación, aunque sus objetivos, contextos o marcos teóricos no coinciden siempre con los de este trabajo. En nuestro caso, la estrategia de recogida de información requiere definir previamente qué esquemas de conocimiento - referenciales de la ciencia escolar-queremos que activen los estudiantes $\mathrm{y}$, a partir de ellos, plantear diferentes situaciones (experiencias, experimentos, cuestiones de papel y lápiz...) para que pongan de manifiesto sus esquemas de razonamiento y de acción en distintos contextos.

\section{A. Determinación de los esquemas referenciales de la ciencia escolar}

Como ya indicamos, hemos elaborado cuatro esquemas de razonamiento y cuatro esquemas de acción. Todos ellos juegan un papel destacado en la descripción, justificación e interpretación de los cambios de un sistema en este dominio.

\begin{tabular}{|c|l|}
\hline \multicolumn{1}{|c|}{ Tópico } & \multicolumn{1}{c|}{ Ejemplos de aportaciones } \\
\hline Temperatura. Equilibrio térmico & $\begin{array}{l}\text { Brook et al. (1984), Driver y Russell (1982), Erikson (1989), Erikson y Tiberghien } \\
\text { (1989), Shayer y Wylam (1981), Stavy y Berkovitz (1980), Strauss (1983), Tiberghien } \\
(1980), \text { Tiberghien y Barboux (1983), Valcárcel et al. (1990) }\end{array}$ \\
\hline Calor y variación de temperatura & $\begin{array}{l}\text { Brook et al. (1984), Erikson (1989), Erikson y Tiberghien (1989), García y Rodríguez } \\
\text { (1985), Hierrezuelo et al. (1987), Shayer y Wylam (1981), Strauss (1983), Summers } \\
(1983), \text { Valcárcel et al. (1990), Vázquez (1987) }\end{array}$ \\
\hline Dilatación & $\begin{array}{l}\text { Domínguez et al., (1998a), Erikson y Tiberghien (1989), Lew y Treagust (1995), Shayer } \\
\text { y Adey (1986), Thompson (1989) }\end{array}$ \\
\hline Cambio de estado & $\begin{array}{l}\text { Driver y Russell (1982), Hierrezuelo y Montero (1989), Tiberguien y Barboux (1983), } \\
\text { Valcárcel et al. (1990) }\end{array}$ \\
\hline Modelo de partículas & $\begin{array}{l}\text { Brook et al. (1984), Benarroch (1998), Benarroch et al. (1999), Domínguez et al. } \\
\text { (1998a), Furió y Hernández (1983), Llorens (1991), Marín y Benarroch (1994), Mitchell } \\
\text { y Kellington (1982), Novick y Nussbaum (1981), Piaget e Inhelder (1982), Posada } \\
\text { (1993), Pozo et al. (1991), Schauble et al. (1991), Shayer y Adey (1986), Stavy (1990) }\end{array}$ \\
\hline
\end{tabular}


Figura 1

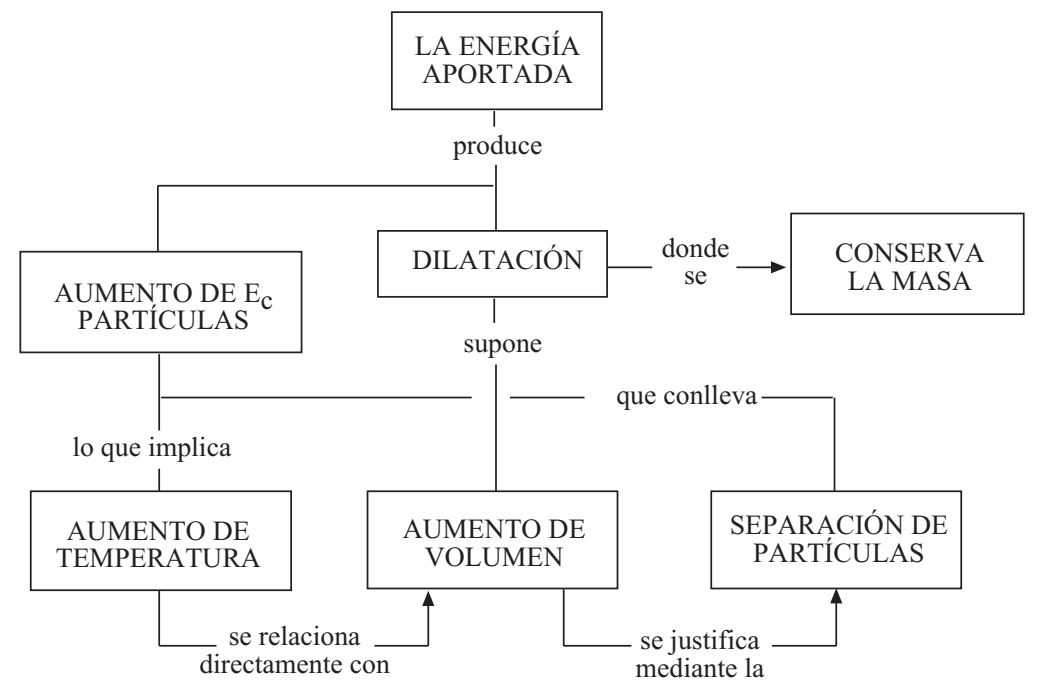

Como nuestra intención es conseguir una representación de la estructura de relaciones establecidas entre el esquema dominante, los subesquemas y las variables, los representaremos mediante etiquetas, con letra negrita los esquemas y subesquemas; y sus relaciones, mediante líneas con nexos que indiquen la naturaleza de la relación. Cuando alguno de estos elementos aparece en el discurso, diremos que el elemento ha sido activado. Lo mismo haremos cuando se trate del discurso, oral o escrito, de los estudiantes.

Por razones de espacio, sólo nos referiremos al esquema de razonamiento dilatación y al de acción relación entre variables.

\section{Esquema de razonamiento Dilatación}

En la figura 1 aparece el esquema de razonamiento Dilatación; de esta manera se hace explícito el conocimiento utilizado, deseable desde la ciencia escolar.

Se activan Dilatación, Aumento de energía cinética de las partículas, Aumento de volumen, Aumento de temperatura y Conserva la masa. Así mismo se activan Energía aportada, asociada a sistema que subyace ausente, y partículas a la que se ha asignado el valor separación. El esquema integra dos tipos de razonamiento -macroscópico y microscópico- para reconocer e interpretar el fenómeno de la dilatación.

Respecto del macroscópico, se ha relacionado el esquema dominante, Dilatación, con Energía aportada y con $\mathrm{Au}-$ mento de volumen, lo que le da significado; el cambio que- da determinado por la relación entre Aumento de temperatura y Aumento de volumen. La relación entre Dilatación y Conserva la masa pone de manifiesto que, durante el proceso, no hay un incremento de la misma.

Respecto del microscópico, se utiliza el modelo cinético de partículas. Para ello, se establecen las relaciones entre Energía aportada, Aumento de la energía cinética de las partículas, Separación de partículas y Aumento de volumen. Así, se pone de manifiesto la relación entre la energía aportada al sistema, el incremento de la energía cinética de las partículas y la separación de las mismas, con el consiguiente incremento de volumen del sistema, y el aumento de la temperatura.

La existencia de subesquemas y variables permite asignarles valores y, consecuentemente, especificar los diferentes papeles que pueden jugar en el esquema; éstos pueden cambiar en función del contexto y del contenido. De este modo un subesquema o una variable pueden cambiar su papel. Por ejemplo, Energía aportada es una variable en los esquemas Dilatación y Relación entre variables. Esto es debido a que, en estos casos, sólo nos interesa si el alumnado hace explícito o no el intercambio de energía, y no cuál es su significado. Pero, en otras circunstancias, podríamos necesitar profundizar en qué concepción tiene el alumnado los términos utilizados.

Además nos permiten generar asignaciones ausentes, cuando la asignación de valores no puede hacerse simplemente tomando como base el estímulo real o recurriendo a la memoria; éstas también son llamadas valores por defecto, pues un esquema puede contener gran cantidad de detalles 
Figura 2

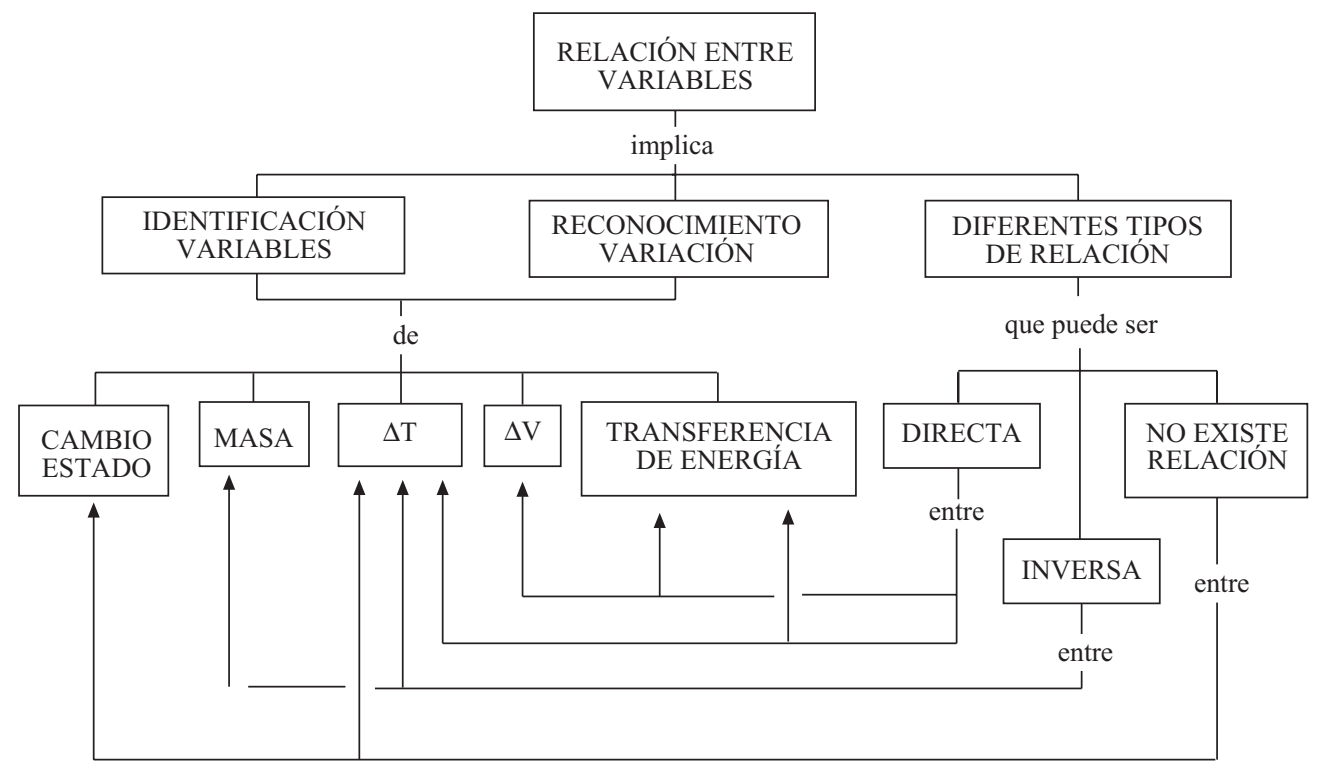

supuestos sin que su existencia esté garantizada. Por ejemplo, se ha hecho la suposición de que, cuando se indica Energía aportada, se le está dando, implícitamente, un valor positivo; es decir, se está suponiendo que es el mechero el que cede energía al sistema.

La capacidad de encaje de los subesquemas en el dominante nos permite representar características importantes de las inferencias; en concreto, el hecho de que tienen estructuras constituyentes. De este modo, aunque Aumento de volumen es un subesquema que representa una inferencia, tiene estructuras complejas que serán distintas en función de las relaciones que establezca con los demás elementos: no es lo mismo que se relacione con Incremento de temperatura, con Dilatación o con Separación de las partículas.

Esquema de acción Relación entre variables

En la figura 2 se ilustra el esquema de acción Relación entre variables. En la parte izquierda del esquema se han activado Identificación de variables y Reconocimiento de variación, que se relacionan con las variables Cambio de estado, Incremento de temperatura e Incremento de volumen, Transferencia de energía y Masa, para indicar que se han identificado y se ha reconocido su variación.

En la parte derecha se activa Diferentes tipos de relación, al que se han dado los valores directa, inversa y no existe relación.
Se establecen relaciones: directas entre Transferencia de energía, Incremento de volumen e Incremento de temperatura; inversa entre Masa e Incremento de temperatura; y no se establece relación entre Cambio de estado e Incremento de temperatura para hacer explícita la constancia de la temperatura en el proceso.

B. Diseño de las pruebas de recogida de información

Definidos los esquemas referenciales de la ciencia escolar, se diseñaron nueve pruebas para la caracterización inicial de la muestra, algunas de las cuales han sido descritas y analizadas en otros trabajos (Domínguez et al., 1998b, 1998-99, 1999, 2000).

Se plantean situaciones experienciales -se realiza una experiencia delante del grupo-y, a partir de ésta, planteábamos a los estudiantes una serie de interrogantes. En otras les pedíamos individualmente que registraran datos con algún instrumento de laboratorio. También, y aprovechando que estaban habituados a realizar mapas conceptuales, hemos utilizado esta técnica para recoger información. Por último, usamos cuestiones de lápiz y papel sobre hechos muy próximos y cotidianos. Creemos que, de esta forma, hemos podido establecer sus esquemas de conocimiento en diferentes contextos e interrelacionados con diversos contenidos. En el cuadro II, por razones de espacio, se recogen una breve descripción y la intención de recogida de información de las pruebas de las que hemos extraído relaciones correspondientes a dilatación y a relación entre variables. 


\begin{tabular}{|c|c|c|}
\hline Prueba & Breve descripción & Qué se quiere investigar \\
\hline 1 & $\begin{array}{l}\text { El profesor realiza la experiencia de elevar la temperatura } \\
\text { de distintos volúmenes de agua contenidos en dos tubos } \\
\text { de ensayo, mediante un mechero de alcohol, que se sitúa } \\
\text { debajo de ambos tubos. } \\
\text { Se pide al alumnado que responda unos ítems sobre lo } \\
\text { ocurrido en la experiencia. }\end{array}$ & $\begin{array}{l}\text { Si asocia una acción externa, la energía aportada por el } \\
\text { mechero, con la causa del incremento de la temperatura } \\
\text { del sistema. } \\
\text { Si reconoce la proporcionalidad inversa entre la masa y el } \\
\text { incremento de temperatura. } \\
\text { Si reconoce el concepto calor en una experiencia real. } \\
\text { Cómo influye en su respuesta la polisemia del vocablo } \\
\text { calentar y otros derivados de la vida diaria. }\end{array}$ \\
\hline 2 & $\begin{array}{l}\text { Se pide que elaboren un mapa conceptual con una lista } \\
\text { cerrada de ocho palabras: temperatura, energía, calor, } \\
\text { cuerpo, agitación, partículas, dilatación y termómetro. }\end{array}$ & $\begin{array}{l}\text { Si hacen explícitas las ideas: } \\
\text { - Macroscópica: los cuerpos tienen energía y la pueden } \\
\text { transferir mediante el proceso denominado calor, si existe } \\
\text { diferencia de temperatura entre ellos. Un cuerpo } \\
\text { experimenta dilatación al elevarse su temperatura. } \\
\text { - Microscópica: un cuerpo está formado por partículas } \\
\text { que, al recibir energía por medio del proceso denominado } \\
\text { calor, incrementan su agitación; en consecuencia, su } \\
\text { temperatura se eleva y el cuerpo experimenta dilatación. }\end{array}$ \\
\hline 5 & $\begin{array}{l}\text { Se presenta el relato de una experiencia (calentamiento } \\
\text { de un recipiente lleno de agua) y una tabla de valores } \\
\text { tiempo/temperatura. } \\
\text { Deben responder cuatro ítems sobre la elaboración de la } \\
\text { gráfica, y la descripción e interpretación de sus tramos. }\end{array}$ & $\begin{array}{l}\text { Cómo realizan una representación gráfica tiempo/ } \\
\text { temperatura, a partir de una tabla de valores. } \\
\text { Si perciben, en la tabla y en la gráfica, que la temperatura } \\
\text { varía con el tiempo y que se mantiene constante a los } \\
100^{\circ} \mathrm{C} \text {. } \\
\text { Cómo justifican la variación y cómo explican que la } \\
\text { temperatura se estabilice al llegar a los } 100^{\circ} \mathrm{C} \text {. }\end{array}$ \\
\hline 6 & $\begin{array}{l}\text { Se presenta una gráfica sobre temperatura/tiempo } \\
\text { con tres ramas: una recta de pendiente positiva, otra con } \\
\text { pendiente cero y una tercera con pendiente positiva. } \\
\text { Se pregunta por el significado de la gráfica y se piden } \\
\text { ejemplos que se puedan relacionar con ella. }\end{array}$ & $\begin{array}{l}\text { Cómo interpretan los estudiantes la representación gráfica. } \\
\text { Qué significado físico asignan a las diferentes ramas de la } \\
\text { misma. } \\
\text { Si son capaces de dar ejemplos de situaciones que puedan } \\
\text { ser representadas con dicha gráfica. }\end{array}$ \\
\hline 7 & $\begin{array}{l}\text { Se describe una experiencia: un globo desinflado en el } \\
\text { cuello del matraz; se calienta el matraz y el globo se } \\
\text { hincha (se ilustra con figuras). } \\
\text { Se pide que dibujen qué ocurre en el interior del matraz } \\
\text { y del globo antes y después de calentar, y que expliquen } \\
\text { las diferencias que han querido representar. }\end{array}$ & $\begin{array}{l}\text { Si reconocen el fenómeno de dilatación de los gases y } \\
\text { cómo lo justifican. } \\
\text { Cómo utilizan el modelo particular en la interpretación } \\
\text { del fenómeno. } \\
\text { Cómo representan la evolución de las partículas en el } \\
\text { proceso. }\end{array}$ \\
\hline 8 & $\begin{array}{l}\text { Se presentan dos dibujos de un cuerpo sólido (uno un } \\
\text { poco mayor que otro). } \\
\text { Se pide que dibujen qué ocurre en su interior cuando está } \\
\text { frío y caliente; además debe explicar las diferencias } \\
\text { representadas. }\end{array}$ & $\begin{array}{l}\text { Cómo utilizan el modelo de partículas para interpretar dos } \\
\text { situaciones diferentes. } \\
\text { Cómo representan las partículas cuando el cuerpo tiene } \\
\text { diferentes temperaturas. }\end{array}$ \\
\hline 9 & $\begin{array}{l}\text { Se describe que, para hacer chocolate caliente en un } \\
\text { desayuno, se utilizan dos cucharas, una de madera y otra } \\
\text { de metal. Se indica que, al cabo de un cierto tiempo, el } \\
\text { mango de metal está caliente y el de madera no. } \\
\text { Se pide al alumnado cómo lo justifican y cómo lo } \\
\text { representan con el modelo de partículas. }\end{array}$ & $\begin{array}{l}\text { Cómo utilizan el modelo de partículas en esta situación. } \\
\text { Cómo representan las partículas cuando un cuerpo aumenta } \\
\text { su temperatura. } \\
\text { Si reconocen la dependencia de la temperatura con el tipo } \\
\text { de material cuando se aporta energía a un sistema por } \\
\text { calentamiento. }\end{array}$ \\
\hline
\end{tabular}




\section{Obtención de los esquemas de los alumnos}

Sin duda, la información recogida con cuestionarios y entrevistas de las características de nuestras pruebas es rica pero también compleja. El objetivo de nuestra investigación es, no sólo el análisis individual de la información recabada en cada ítem, sino inferir y hacer explícitos los esquemas de conocimiento que utilizan y activan los estudiantes cuando se les plantean nueve situaciones diferentes.

Para ello, hemos fragmentado el discurso -identificación de los subesquemas, de las variables y de sus relacionesde cada uno de los estudiantes en todas y cada una de las pruebas. De esta manera se obtiene el catálogo de todas las afirmaciones usadas por cada alumno en las diferentes situaciones planteadas. Cuando una idea (en forma de proposición) es utilizada varias veces en distintos contextos podemos considerar que no es fruto de la casualidad sino que la misma está suficientemente interiorizada y, por lo tanto, forma parte de un esquema.

Una vez que obtuvimos estas relaciones, las hemos agrupado en función de los esquemas de referencia (Dilatación... Relación entre variables...) y posteriormente hemos construido los esquemas de razonamiento y de acción de los estudiantes. Estos pasos los podremos ver más claramente en el siguiente apartado.

Ahora bien, hemos de señalar que los esquemas que activan los estudiantes son idiosincrásicos y dependen, no sólo del estímulo, sino también del contexto y del contenido. Esto puede dar lugar a que alguno utilice «aparentemente» distintos esquemas de razonamiento y de acción. Así, por ejemplo, cuando utilizan el modelo cinético de partículas para explicar la dilatación de un sólido, pueden adjudicar a Partículas el valor separación y justificar el fenómeno por el incremento de la distancia entre las partículas; sin embargo, en el caso de un globo lleno de aire que se dilata, pueden activar la variable choques para establecer la relación causal entre la dilatación del globo y los choques de las partículas contra las paredes del mismo.

Esto no constituye un problema en nuestra investigación, ya que podemos suponer que el alumno o la alumna han construido esquemas «más amplios»-integrando otros complementarios- aunque no siempre los active de forma completa. La situación es más compleja de justificar cuando utiliza «esquemas contradictorios» en función del contexto; en estos casos, hemos optado por decir que no podemos identificar el esquema de conocimiento.

\section{RESULTADOS OBTENIDOS}

Como indicábamos, hemos «troceado» todas las afirmaciones contenidas en las respuestas de los alumnos y alumnas en cada ítem y en cada prueba. Una vez agrupadas en función de los diferentes esquemas, hemos diferenciado las relaciones que coincidían con las de los esquemas referenciales y las que eran contradictorias o no aparecían.
También hemos representado gráficamente los esquemas de razonamiento y de acción, siguiendo el mismo procedimiento que el utilizado para la elaboración de los esquemas referenciales. Las etiquetas ilustradas con trama de puntos indican que no coinciden algunos subesquemas o variables con lo deseable desde la ciencia escolar. Cuando las relaciones sean las no coincidentes, se ha indicado utilizando letra cursiva en los nexos.

Una vez identificados, es posible analizarlos, discutirlos, compararlos y ordenarlos según su potencialidad explicativa, interpretativa o predictiva, su proximidad al deseable, etc. Además nos permite contrastar la evolución en el conocimiento de cada estudiante en los diferentes tempus de la investigación -antes, durante y después de nuestra intervención educativa- aunque no nos ocupemos de ello en este trabajo.

Ejemplificaremos el proceso para los esquemas de los que nos hemos ocupado anteriormente: Dilatación y Relación entre variables

\section{Esquemas de razonamiento de los estudiantes: Dilata- ción}

Identificadas las relaciones entre esquema dominante, subesquemas y variables, que constituyen el razonamiento utilizado por los estudiantes en sus respuestas, se han recogido en la tabla I sus frecuencias correspondientes $(f)$, donde puede verse que algunas relaciones son activadas reiteradamente.

Destaca el reconocimiento de la relación causal entre el calor aportado al sistema y la dilatación del mismo o de

Tabla I

Relaciones extraídas de las pruebas.

\begin{tabular}{|l|c|}
\hline \multicolumn{1}{|c|}{ RELACIONES } & f \\
\hline Calor produce dilatación & 51 \\
\hline Dilatación => Mayor volumen & 10 \\
\hline Dilatación => Aumento de la temperatura & 39 \\
\hline Calor $=>$ (Dilatación) => Movimiento de las partículas & 13 \\
\hline Dilatación $=>$ Separación de las partículas & 10 \\
\hline Dilatación $=>$ Cambio de ubicación de la sustancia & 26 \\
\hline Dilatación $=>$ Disminución del peso de la sustancia & 5 \\
\hline Dilatación $=>$ Disminución de la densidad de la sustancia & 2 \\
\hline Dilatación $=>$ Aumento de la cantidad de la sustancia & 3 \\
\hline Dilatación $=>$ Aumento del tamaño de las partículas & 9 \\
\hline Dilatación $=>$ Cambio de la sustancia & 1 \\
\hline
\end{tabular}




\section{INVESTIGACIÓN DIDÁCTICA}

Tabla II

Pruebas de las que se han extraído las relaciones sobre dilatación.

\begin{tabular}{|l|c|}
\hline \multicolumn{1}{|c|}{ RELACIONES } & f \\
\hline Prueba 2 & 14 \\
\hline Prueba 5 & 1 \\
\hline Prueba 7 & 17 \\
\hline Prueba 8 & 18 \\
\hline Prueba 9 & 5 \\
\hline$f=$ número de veces que se han activado las relaciones & \\
\hline
\end{tabular}

que este fenómeno implica un incremento de temperatura. Estos logros -que nos reafirman en la creencia de que nuestros alumnos y alumnas «saben algo»...-, podremos aprovecharlos en la intervención en el aula, aunque trataremos de que calor sea conceptualizado como transferencia de energía.

Lógicamente también hay que señalar relaciones que no son deseables desde la ciencia escolar: en el caso de los gases, asocian la dilatación -para el caso de un globo acoplado al cuello de un matraz que calentamos con un mechero- con el cambio de ubicación de la sustancia que cons- tituye el sistema, explicando que el aire del matraz pasa al globo. Algunos justifican el fenómeno por un incremento del tamaño de las partículas o cambios en la masa y en el peso de la sustancia.

En la tabla II se recogen las pruebas de las que se han extraído las relaciones. Con esta información queremos resaltar algo en lo que hemos insistido: la activación de un determinado esquema depende del alumno y del contexto.

Así, con estos datos, se puede inferir la mayor o menor idoneidad de algunas pruebas para la activación del esquema Dilatación pero no la garantía de que se active; además, también podemos apreciar que «otros estímulos» (por ejemplo, la prueba 9) imprevisiblemente también los ponen en juego.

No obstante, los objetivos de nuestro trabajo requieren identificar los esquemas de razonamiento individuales de los estudiantes. Por ello, en la tabla III, se han recogido las relaciones activadas por cada uno de ellos (identificados, del 1 al 20, en la fila Referencia de alumnado) distinguiendo las adecuadas y las alejadas de la ciencia escolar.

En función de la adecuación de las relaciones al referencial correspondiente, hemos ordenado los esquemas de razonamiento y se han podido establecer 4 niveles y 6 subniveles con las respuestas de los 20 estudiantes (primera fila de la tabla III). El nivel i4 es el más cercano a lo deseable desde la ciencia escolar y el nivel il es el más lejano de los es-

Tabla III

Esquemas de razonamiento Dilatación: relaciones y niveles.

\begin{tabular}{|c|c|c|c|c|c|c|c|c|c|c|c|c|c|c|c|c|c|c|}
\hline Niveles $\quad \rightarrow$ & $i 4$ & $i 3 a$ & $i 3 b$ & $i 3 c$ & $i 3 d$ & $i 2 a$ & $i 2 b$ & \multicolumn{11}{|c|}{$i 1$} \\
\hline Referencia de alumnado $\rightarrow$ & 8 & 14 & 9,17 & 16 & 15 & 7 & 6 & 1 & 4 & 2 & 10 & 12 & 13,20 & 3 & 11 & 19 & 21 & 18 \\
\hline \multicolumn{19}{|l|}{ Relaciones adecuadas desde la ciencia escolar } \\
\hline Calor produce dilatación & $\mathbf{x}$ & $\mathbf{x}$ & $\mathbf{x}$ & $\mathbf{x}$ & $\mathbf{x}$ & $\mathbf{x}$ & $\mathbf{x}$ & $\mathbf{x}$ & $\mathbf{x}$ & $\mathbf{x}$ & $\mathbf{x}$ & $\mathbf{x}$ & $\mathbf{x}$ & $\mathbf{x}$ & $\mathbf{x}$ & $\mathbf{x}$ & $\mathbf{x}$ & $\mathbf{x}$ \\
\hline Dilatación $=>$ aumento de volumen & $\mathbf{x}$ & $\mathbf{x}$ & $\mathbf{x}$ & $\mathbf{x}$ & & $\mathbf{x}$ & $\mathbf{x}$ & $\mathbf{x}$ & $\mathbf{x}$ & $\mathbf{x}$ & $\mathbf{x}$ & & & & & & & \\
\hline Calor produce movimiento de las partículas & $\mathbf{x}$ & $\mathbf{x}$ & $\mathbf{x}$ & $\mathbf{x}$ & $\mathbf{x}$ & & & $\mathbf{x}$ & $\mathbf{x}$ & & & & & & & & & \\
\hline Dilatación $=>$ separación de las partículas & $\mathbf{x}$ & $\mathbf{x}$ & & & & & & & & $\mathbf{x}$ & & & $\mathbf{x}$ & $\mathbf{x}$ & & & & \\
\hline Dilatación se relaciona con $\Delta \mathrm{T}$ & $\mathbf{x}$ & $\mathbf{x}$ & $\mathbf{x}$ & $\mathbf{x}$ & $\mathbf{x}$ & $\mathbf{x}$ & $\mathbf{x}$ & $\mathbf{x}$ & $\mathbf{x}$ & $\mathbf{x}$ & $\mathbf{x}$ & $\mathbf{x}$ & $\mathbf{x}$ & $\mathbf{x}$ & $\mathbf{x}$ & & $\mathbf{x}$ & \\
\hline \multicolumn{19}{|l|}{ Relaciones alejadas de la ciencia escolar } \\
\hline Dilatación $\Rightarrow>$ cambia ubicación de la sustancia & & $\mathbf{x}$ & $\mathbf{x}$ & $\mathbf{x}$ & $\mathbf{x}$ & $\mathbf{x}$ & $\mathbf{x}$ & $\mathbf{x}$ & $\mathbf{x}$ & $\mathbf{x}$ & $\mathbf{x}$ & $\mathbf{x}$ & $\mathbf{x}$ & $\mathbf{x}$ & $\mathbf{x}$ & & $\mathbf{x}$ & \\
\hline Dilatación $=>$ disminuye el peso & & & & $\mathbf{x}$ & $\mathbf{x}$ & & $\mathbf{x}$ & & & & & & $\mathbf{x}$ & & & & $\mathbf{x}$ & \\
\hline Dilatación $=>$ las partículas se dilatan & & & & & & & & $\mathbf{x}$ & $\mathbf{x}$ & $\mathbf{x}$ & $\mathbf{x}$ & $\mathbf{x}$ & $\mathbf{x}$ & $\mathbf{x}$ & & $\mathbf{x}$ & $\mathbf{x}$ & \\
\hline Dilatación $=>$ aumenta el núm. de partículas & & & & & & & & & & & & & & & & & & $\mathbf{x}$ \\
\hline Dilatación $\Rightarrow>$ aumenta la masa & & & & & & & & & & & $\mathbf{x}$ & & & & & & & \\
\hline Dilatación $\Rightarrow>$ cambia la naturaleza de la sustancia & & & & & & & & & & & & & & & $\mathbf{x}$ & & & \\
\hline
\end{tabular}


quemas correspondientes a la caracterización inicial de la muestra. Cuando dos o más alumnos o alumnas tienen esquemas de conocimiento semejantes o con diferencias poco significativas, los incluimos en el mismo nivel aunque les asignamos distintos subniveles.

En el nivel i4 se establecen las relaciones deseables.

En el nivel i3 se han categorizado esquemas en los que se ha establecido la relación causal entre el cambio de ubicación de la sustancia y el fenómeno de la dilatación. Relaciones deseables y que no lo son justifican la diferenciación en distintos subniveles.

En el nivel i2, no se han activado Movimiento de las partículas ni Separación de las partículas, por lo que no se hace uso del modelo cinético de partículas en la interpretación del fenómeno. Se han diferenciado dos subniveles.

A partir de las relaciones de la tabla III, hemos realizado la representación de los esquemas. Como ejemplo se ilustran los correspondientes al nivel i2, en el que, como indicamos, se han diferenciado (Fig. 3) dos subniveles: i2a, i2b.

Nivel i2 del esquema de razonamiento sobre Dilatación

Subnivel i2a (alumna 7): Aunque el esquema permite una descripción completa de la dilatación, ésta se justifica, para el caso de los gases, por un cambio de ubicación de las partículas.

Subnivel i2b (alumna 6): Se incrementa el carácter alternativo del razonamiento, pues se considera que el aire ha de disminuir de peso para que pueda ocurrir el cambio de ubicación del mismo.
Nivel il del esquema de razonamiento sobre Dilatación

Se han incluido en este nivel los esquemas más alejados del referencial. Se ilustran los ejemplos del alumno 11 y de alumna 21. (Fig. 4).

En el caso del alumno 11 se considera que la dilatación está relacionada con el cambio de ubicación de las partículas, pero además con el cambio de la naturaleza de la sustancia que constituye el sistema.

La alumna 21 ha asignado a partículas los valores tamaño y número, mediante los que interpreta la dilatación como un incremento del número de partículas que lo constituyen y el aumento de tamaño de las mismas (atribuyen a las partículas características y cambios del sistema).

Esquemas de acción de los estudiantes: Relación entre variables

En este apartado se describen y analizan los esquemas de acción relativos a la relación entre variables implicadas en los cambios del sistema cuando recibe energía desde la fuente. En la tabla IV se recogen las relaciones y sus correspondientes frecuencias de activación.

Se observa que los estudiantes no tienen especiales dificultades para identificar variables y percibir que pueden tomar varios valores (sobre todo, en los casos de transferencia de calor e incremento de temperatura del sistema); sin embargo, se reduce significativamente el reconocimiento del tipo de relación entre ellas.

Las relaciones con las que hemos elaborado los esquemas, las hemos extraído de la prueba 1 (76 relaciones), de la

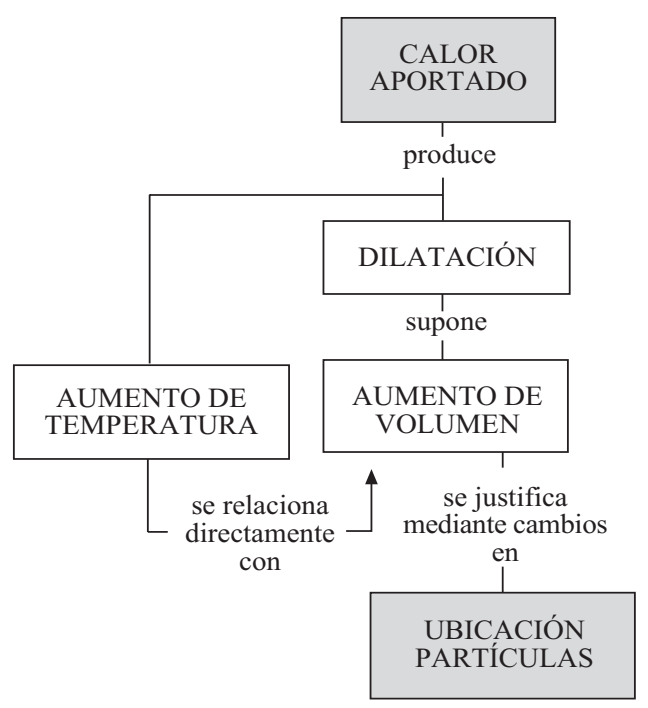

Subnivel i2a

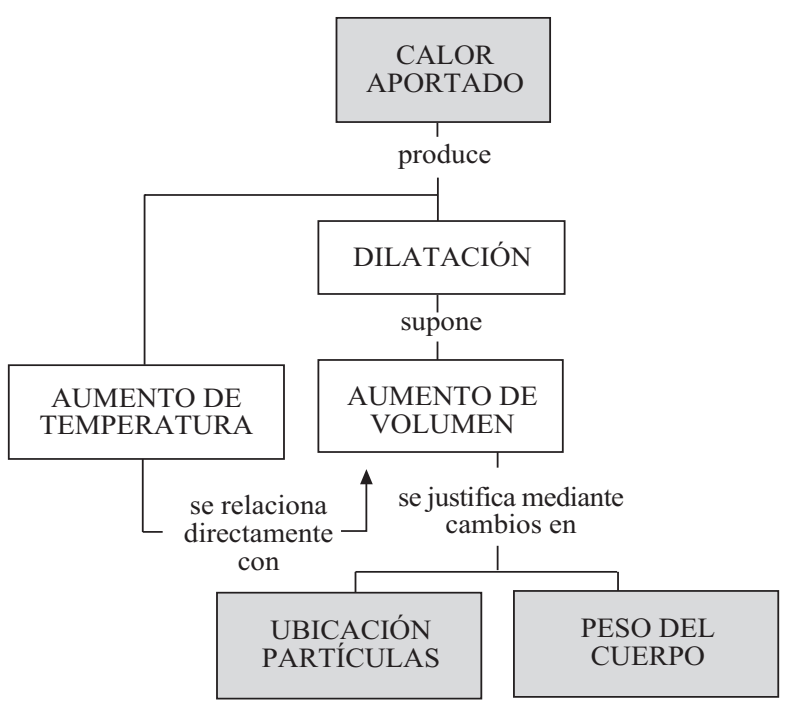

Subnivel i2b 
Figura 4

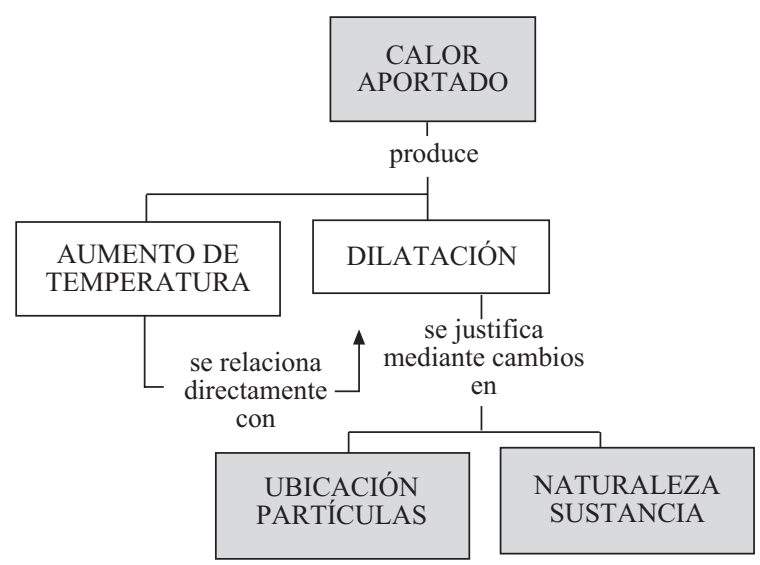

prueba 5 (58), de la prueba 7 (19) y de las pruebas 2 y 8 (10).

En la tabla V hemos recogido las acciones deseables desde la ciencia escolar; se entiende que, cuando no está señalada ésta, no se ha realizado o se ha hecho de forma inadecuada. Obviamente las respuestas del alumnado contienen relaciones entre subesquemas y variables que constituyen esquemas de acción Relación entre variables, que nos han permitido diferenciar cuatro niveles y cuatro subniveles.

Al igual que hemos hecho para los de razonamiento, vamos a comentar brevemente algunos de los esquemas de acción.

Nivel i3 del esquema de acción: Relación entre variables

Los alumnos y alumnas que hemos incluido en este nivel tienen esquemas próximos al referencial de la ciencia escolar. Han relacionado el esquema dominante Relación entre variables con Identificación de variables y Reconocimiento de la variación y éstos, a su vez, los han puesto en relación con Incremento de temperatura y Masa, deseables. Trasferencia de calor (su etiqueta está sombreada por no ser adecuada) deberá ser atendida en nuestra intervención y sustituida por trasferencia de energía. Además, si observamos la parte derecha de la representación, también han reconocido qué tipo de relación se establece; esto se observa en la asociación entre Diferentes tipos de relación y directa e inversa. Identificamos dos subniveles (Figs. $5,6)$ : i3a, i3b.

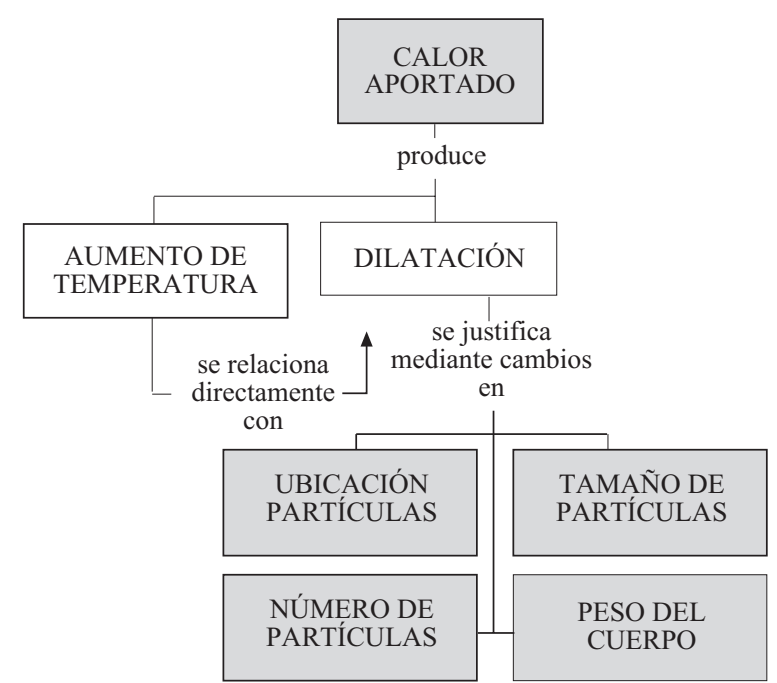

Nivel il

\begin{tabular}{|c|c|}
\hline RELACIONES & $\mathrm{f}$ \\
\hline \multicolumn{2}{|l|}{ Identificación y variación de las variables } \\
\hline Transferencia de calor & 55 \\
\hline Incremento de temperatura & 93 \\
\hline Incremento de volumen & 17 \\
\hline Cambio de estado & 35 \\
\hline Masa & 29 \\
\hline \multicolumn{2}{|l|}{ Directas } \\
\hline Entre transferencia de calor e incremento de temperatura & 8 \\
\hline Entre transferencia de calor e incremento de volumen & 17 \\
\hline \multicolumn{2}{|l|}{ Inversas } \\
\hline Entre masa e incremento de temperatura & 17 \\
\hline \multicolumn{2}{|l|}{ No existe relación } \\
\hline Entre cambio de estado e incremento de temperatura & 22 \\
\hline
\end{tabular}


Tabla V

Esquemas de acción Relación entre variables: relaciones y niveles de orden.

\begin{tabular}{|c|c|c|c|c|c|c|c|c|c|c|}
\hline Niveles $\rightarrow$ & $\mathrm{i} 4$ & i3a & $\mathrm{i} 3 \mathrm{~b}$ & $\mathrm{i} 2 \mathrm{a}$ & $\mathrm{i} 2 \mathrm{~b}$ & \multicolumn{5}{|c|}{ i1 } \\
\hline Referencia del alumnado $\rightarrow$ & 16 & $13,20,21$ & $2,4,6$ & $1,7,8,9,10,14$ & 18 & 17 & 3 & 19 & 11,15 & 12 \\
\hline \multicolumn{11}{|l|}{ Identificación y variación de las variables } \\
\hline Transferencia de calor & $\mathrm{x}$ & $\mathrm{x}$ & $\mathrm{x}$ & $\mathrm{x}$ & $\mathrm{x}$ & $\mathrm{x}$ & $\mathrm{x}$ & $\mathrm{x}$ & $\mathrm{x}$ & \\
\hline Incremento de temperatura & $\mathrm{x}$ & $\mathrm{x}$ & $\mathrm{x}$ & $\mathrm{x}$ & $\mathrm{x}$ & $\mathrm{x}$ & $\mathrm{x}$ & $\mathrm{x}$ & $\mathrm{x}$ & $\mathrm{x}$ \\
\hline Incremento de volumen & $\mathrm{x}$ & & & $\mathrm{x}$ & & $\mathrm{x}$ & & & & \\
\hline Cambio de estado & $\mathrm{x}$ & $\mathrm{x}$ & & & & & $\mathrm{x}$ & $\mathrm{x}$ & $\mathrm{x}$ & \\
\hline Masa (cantidad de agua) & $\mathrm{x}$ & $\mathrm{x}$ & $\mathrm{x}$ & $\mathrm{x}$ & $\mathrm{x}$ & & $\mathrm{x}$ & $\mathrm{x}$ & $\mathrm{x}$ & $\mathrm{x}$ \\
\hline \multicolumn{11}{|l|}{ Relaciones directas } \\
\hline Entre transferencia de calor e incremento de $\mathrm{T}$ & $\mathrm{x}$ & $\mathrm{x}$ & $\mathrm{x}$ & & $\mathrm{x}$ & & & & & \\
\hline Entre transferencia de calor e incremento de V & $\mathrm{x}$ & & $\mathrm{x}$ & $\mathrm{x}$ & & $\mathrm{x}$ & & & & \\
\hline \multicolumn{11}{|l|}{ Relaciones inversas } \\
\hline Entre masa e incremento de temperatura & $\mathrm{x}$ & $\mathrm{x}$ & $\mathrm{x}$ & $\mathrm{x}$ & $\mathrm{x}$ & & $\mathrm{x}$ & $\mathrm{x}$ & & $\mathrm{x}$ \\
\hline \multicolumn{11}{|l|}{ No existe relación } \\
\hline Cambio de estado e incremento de temperatura & $\mathrm{x}$ & $\mathrm{x}$ & & & & & $\mathrm{x}$ & & & \\
\hline
\end{tabular}

Figura 5

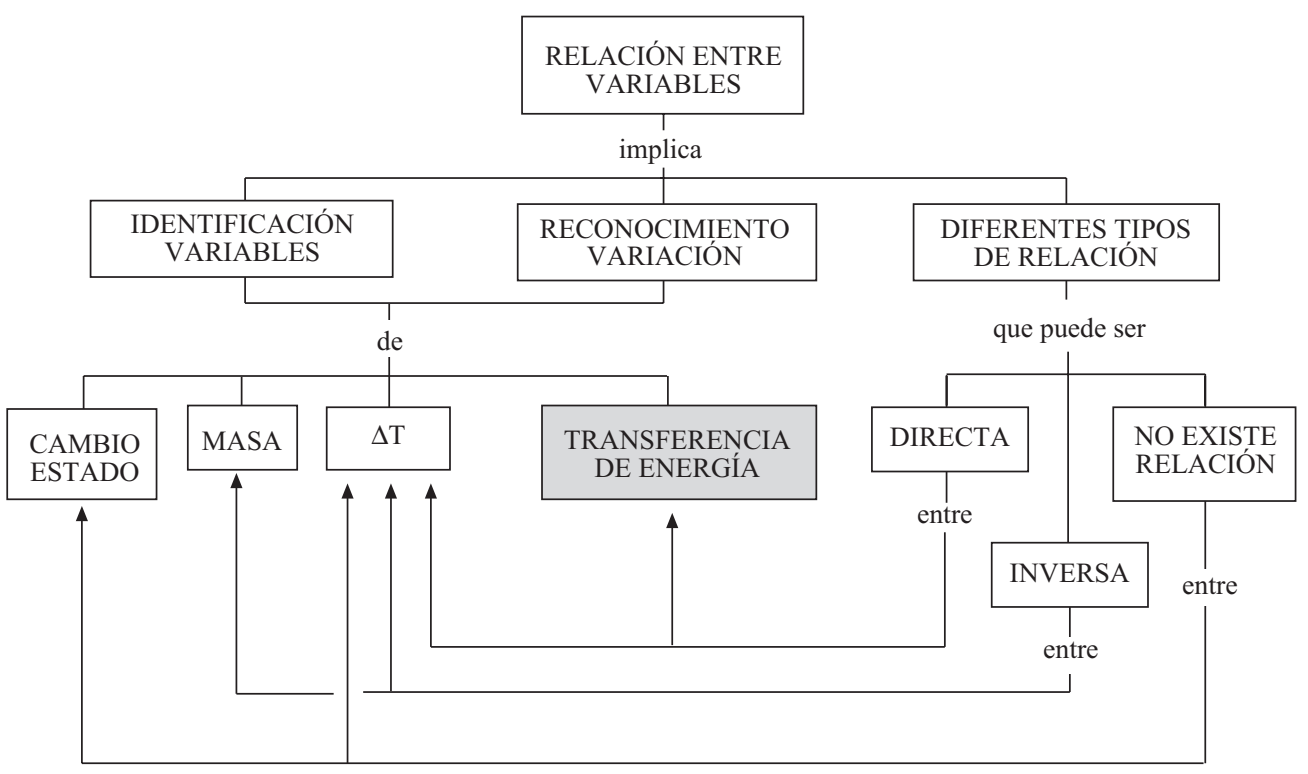

Subnivel i3a 
Figura 6

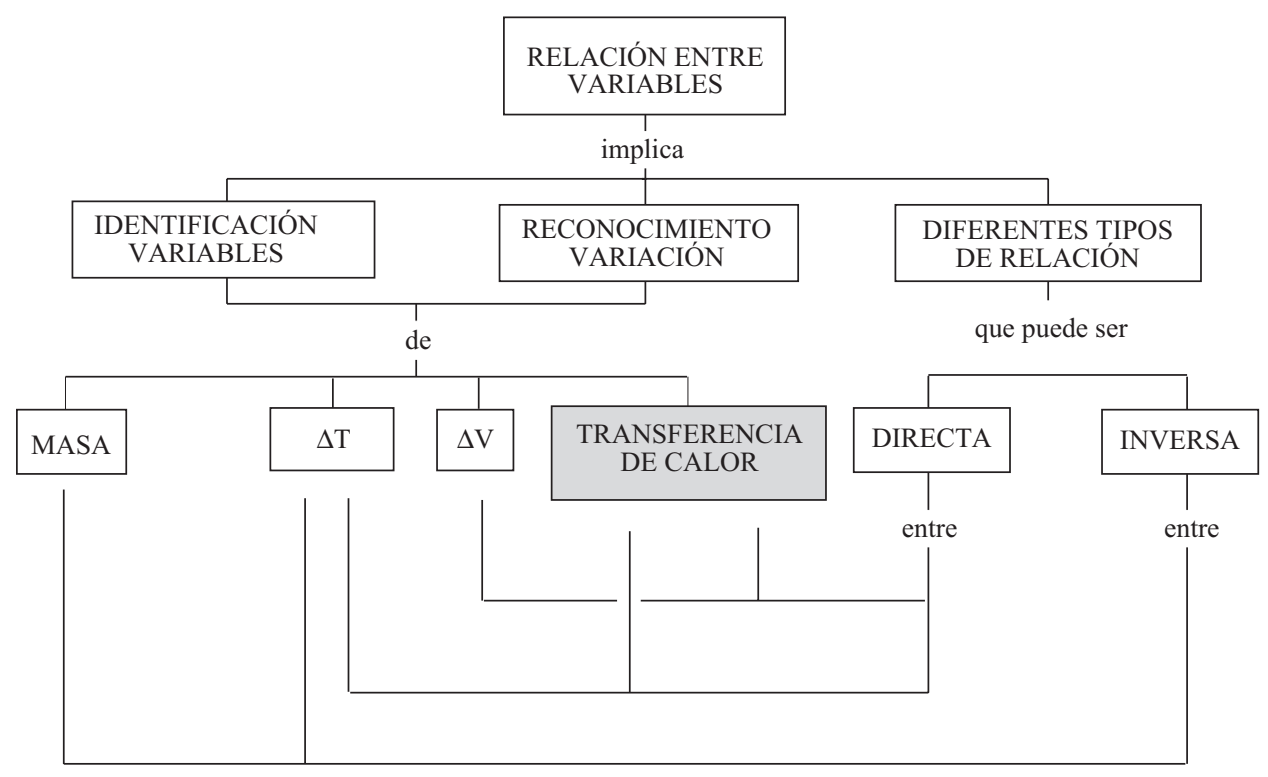

Subnivel i3b

Figura 7

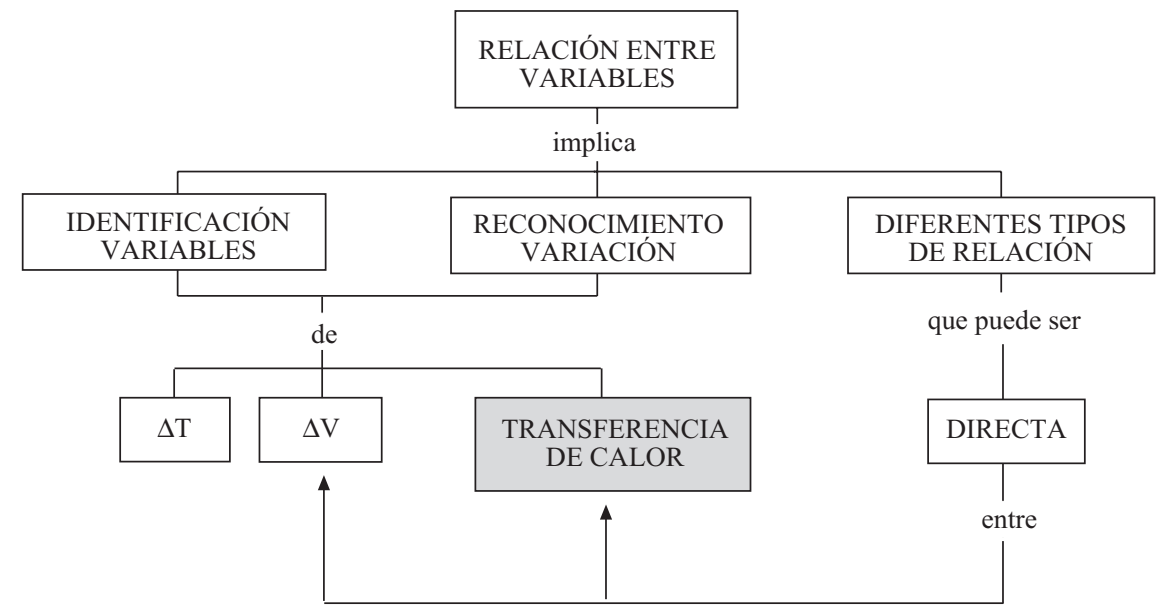

Nivel il

Subnivel i3a (alumnas 13, 20 y 21): No activan Incremento de volumen y, en consecuencia, tampoco su relación con Trasferencia de calor.
Subnivel i3b (alumno 2 y alumnas 4 y 6): No identifican Cambio de estado ni que en el mismo la temperatura no cambia, pues no existe relación con Incremento de temperatura. 
Nivel il del esquema de acción: Relación entre variables

En este nivel hemos incluido aquellos esquemas más alejados de la ciencia escolar. Se representa (Fig. 7) el esquema de la alumna 17.

\section{CONCLUSIONES E IMPLICACIONES DIDÁC- TICAS}

Para establecer las conclusiones vamos a distinguir las que se refieren a los esquemas de razonamiento y acción -como indicábamos, nos centraremos sólo en los de Dilatación y Relación entre variables - y las relacionadas con la estrategia de investigación utilizada.

\section{A. Significado y valores de los esquemas de cono- cimiento}

En primer lugar, podemos decir que, a la vista de los resultados, hay que aceptar la hipótesis que planteábamos: los conocimientos iniciales (conceptuales y procedimentales) de los alumnos de nuestra investigación parecen limitados, insuficientes y, desde luego, alejados del punto de vista de la ciencia escolar.

No obstante, podemos considerar positivo el hecho de que, a menudo, los estudiantes han activado unos esquemas incipientes -sencillos, incompletos y discutibles-que les han permitido interpretar los cambios producidos en un sistema material por un aporte de energía, lo que habla en favor de la influencia de un aprendizaje anterior (sea escolar o extraescolar).

Por otro lado, se detecta que, en muchos momentos, han usado esquemas complementarios (probablemente activan sólo una parte de otro supuestamente más amplio) o contradictorios. Esto puede ser una consecuencia de la falta de estabilidad y consistencia de los mismos o un indicador de la dependencia del contenido y del contexto. En cualquier caso, el hecho de no coincidir con los referenciales de la ciencia escolar que definimos nos exige una intervención intencionada que permita la evolución de los esquemas.

Centrándonos en los resultados obtenidos, se establecen las siguientes conclusiones respecto de los dominios particulares sobre los que nos hemos ocupado en este trabajo.

\section{Esquemas de razonamiento: Dilatación}

Los alumnos y alumnas han establecido relaciones que resultan próximas al punto de vista de la ciencia escolar, pero también otras bastante alejadas. Así se puso de manifiesto cuando, ante un fenómeno de dilatación de gases (prueba del globo y del matraz), lo justificaban porque todo o la mayor parte del aire había cambiado de lugar o de ubicación; o, al interpretar el fenómeno de la dilatación de un sólido, consideraban que las partículas que lo constituyen aumentan de tamaño o que aumenta la cantidad de partículas. Creemos que estas situaciones demandan no sólo la inclusión de nuevas estrategias didácticas sino un cambio profundo en la selección del contenido de enseñanza; por ejemplo, introduciendo el modelo cinético de partículas.

La noción fundamental -materia discontinua y compuesta por partículas- es de primordial importancia para la explicación causal de este tipo de cambios en un sistema material. Pensamos que sólo así podrán estructurar un razonamiento que les aleje de sus creencias -alternativas pero con un cierto grado de coherencia- y éstas les permitan explicar, justificar e interpretar hechos que les son planteados desde la ciencia escolar. De esta manera, para construir el significado de la dilatación, se relacionaría la transferencia de energía al sistema con el aumento de la velocidad de las partículas; se produciría la separación de las mismas y, como consecuencia de ello, un incremento de volumen del sistema.

\section{Esquemas de acción: Relación entre variables}

Un número significativo de estudiantes no ha tenido grandes problemas para identificar el incremento de temperatura, el incremento de volumen, la masa... en los procesos reales o representados que hemos utilizado. No estamos tan seguros de que sean realmente conscientes de que el atributo asociado a una variable es precisamente su variabilidad; es decir, la conciencia de que puede tomar diferentes valores. Pero, donde se han dado los resultados más preocupantes ha sido en el establecimiento de relaciones entre variables.

En muchos casos, no han sido capaces de identificar la relación directa entre la energía transferida y el incremento de temperatura (probablemente porque no diferencian calor y temperatura) o de volumen (no son conscientes o no llegan a hacer explícita esta relación causal). También es elevado el número de los que no han señalado que la temperatura no varía cuando tiene lugar el cambio de estado; menos problemas parecen existir para reconocer la proporcionalidad inversa entre el incremento de temperatura y la masa del sistema ante un determinado aporte de energía al mismo.

Hemos de señalar que los resultados de nuestra investigación (Domínguez, 2000), respecto del aprendizaje de los procedimientos, ponen de manifiesto que inicialmente los estudiantes no sólo tenían serias dificultades para establecer relaciones entre variables, sino que también tenían problemas con la medición, la representación de datos o el diseño experimental. ¿Se los habrán enseñado a lo largo de los siete años que llevaban sumergidos en la educación obligatoria? Se reafirma nuestra opinión de que -aunque se suele encontrar una cierta unanimidad en el profesorado para admitir que los contenidos procedimentales poseen un alto valor formativo para la enseñanza obligatoria de un ciudadano o son imprescindibles en el aprendizaje de las ciencias- no siempre estas opiniones tan mayoritarias se proyectan en la «selección real» del contenido, en el diseño de las actividades o en las intervenciones que se realizan en las aulas (Pro, 1999; Pro et al., 1999, 2000).

Por lo tanto, es preciso diseñar actividades específicas -escalonadas según su grado de dificultad- para enseñar este tipo de contenidos procedimentales. Así, en nuestra 
propuesta de enseñanza, además de incidir en los conceptos y modelos que consideremos adecuados, hemos puesto énfasis en el estudio de los procedimientos más utilizados para elevar la temperatura de los cuerpos. Se relaciona la variación de temperatura del sistema con la transferencia de energía; se da significado al concepto de equilibrio térmico; se construye el significado de calor como proceso de transferencia de energía entre sistemas a diferente temperatura; y se establece la relación entre la variación de temperatura del sistema y la masa y naturaleza de la sustancia que lo constituye.

\section{B. Los esquemas como instrumento de análisis del co- nocimiento}

Lo primero que quisiéramos resaltar es que la estrategia de investigación utilizada nos ha permitido dimensionar el conocimiento y, de esta manera, hacer explícitos los esquemas activados por nuestros alumnos y alumnas. Esto ha hecho posible un mejor conocimiento de qué saben y piensan, lo que consideramos muy positivo, tanto desde el punto de vista docente como investigador. Pero, además, hemos recogido una información a la que probablemente no hubiéramos podido acceder con un análisis clásico de las contestaciones realizadas en cada uno de los ítems de las pruebas, tan habitual en algunos estudios diagnósticos.

A la vista de los resultados de nuestra investigación podemos afirmar que las respuestas del alumnado contienen relaciones que se repiten con una cierta regularidad en diferentes situaciones, que constituyen los que hemos denominado esquemas de razonamiento y de acción. Son esquemas incipientes -gérmenes en la denominación de Vygotski (1979)- que, como docentes, hemos de aprovechar para trabajar en sus ZDP (zonas de desarrollo próximo) para construir y estructurar el nuevo conocimiento.

Ante los estímulos que suponen las pruebas planteadas, los estudiantes han activado los subesquemas, las variables y sus relaciones, que forman parte de las estructuras

\section{REFERENCIAS BIBLIOGRÁFICAS}

BANDIERA, M., CARAVITA, S., TORRACCA, E. y VICENTINI, M. (eds.) (1999). Research in Science Education in Europe. Londres: Kluwer Academic Publishers.

BENARROCH, A. (1998). «Las explicaciones de los estudiantes sobre las manifestaciones corpusculares de la materia. Descripción, análisis y predicción de características y dificultades». Tesis doctoral inédita. Universidad de Granada.

BENARROCH, A., MARÍN, N. y PERALES, J. (1999). La evolución del conocimiento sobre la naturaleza corpuscular de la materia, en Martínez Losada, C. y García Barros, S. (eds.). de su conocimiento. La existencia de similares y distintas formas de pensar o actuar se refleja en la similitud o en la heterogeneidad de los esquemas de razonamiento y de acción iniciales que hemos identificado.

Los diferentes valores que asignan a los subesquemas y a las variables, así como los diversos tipos de relaciones que establecen entre ellos, dan lugar a razonamientos y acciones diferentes. Estas diferencias nos han permitido, incluso, ordenar los esquemas en niveles, en función de su proximidad o lejanía a los esquemas referenciales. Por otro lado, los subniveles nos han servido para diferenciar, en cada nivel, esquemas muy similares o con diferencias poco significativas respecto al aprendizaje.

Aunque no es objeto de este trabajo, la posibilidad de ordenarlos ha facilitado la tarea de estudiar la evolución que se produce en el aprendizaje de cada estudiante, antes, durante y después de la aplicación de la propuesta didáctica. Esto ha permitido conocer qué cambios se han producido individualmente con nuestra intervención, con qué actividades se han favorecido las mayores modificaciones en los esquemas originales, qué estructuras de conocimiento son estables y se mantienen después de un cierto tiempo...

En definitiva, la estrategia de identificación de los esquemas de razonamiento y de acción nos ha permitido acceder a un mejor y más adecuado conocimiento de nuestros alumnos y alumnas, lo que permite aprovechar sus potencialidades.

\section{AGRADECIMIENTOS}

Al Ministerio de Ciencia y Tecnología (MCyT), la financiación (70\% fondos FEDER) del proyecto (BSO2002-04073-C02-02) «Desarrollo de competencias comunicativas y aprendizaje de las ciencias: lenguaje, operaciones epistémicas y uso de modelos».

La didáctica de las ciencias. Tendencias actuales. La Coruña: Servicio de Publicacións da Universidade da Coruña.

BROOK, A., BRIGGS, H., BELL, B. y DRIVER, R. (1984). Aspects of Secondary Students' Understanding of Heat: Full Report. CLIS: Children's Learning in Science Project. Londres: Centre for Studies in Science and Mathematics Education. Universidad de Leeds.

DOMÍNGUEZ, J.M. (2000). Evolución de las formas de hacer y de pensar sobre un sistema material, en el marco de la termodinámica y del modelo de partículas. Estudio mediante es- 
quemas de acción y de razonamiento. Santiago de Compostela: Servicio de Publicaciones de la Universidad de Santiago de Compostela.

DOMÍNGUEZ, J.M., PRO, A. y GARCÍA-RODEJA, E. (1998a). Las partículas de la materia y su utilización en el campo conceptual de calor y temperatura: un estudio transversal. Enseñanza de las Ciencias, 16(3), pp. 461-475.

DOMÍNGUEZ J.M., PRO, A., GARCÍA-RODEJA, E. y ILLOBRE, M.L. (1998b). Esquemas conceptuales de los alumnos de secundaria sobre el modelo de partículas de la materia, en Banet, E. y Pro, A. Investigación e innovación en la enseñanza de las ciencias, vol. II. Lleida: DM.

DOMÍNGUEZ, J.M., PRO, A., GARCÍA-RODEJA, E. y ILLOBRE, M.L. (1998-99). Os esquemas de acción e a avaliación do coñecemento procedimental. A medida da temperatura na ESO. ADAXE. Revista de Estudios e Experiencias Educativas, 14-15, pp. 233-270. Facultade de Ciencias da Educación. Universidade de Santiago de Compostela.

DOMÍNGUEZ J.M., PRO, A., GARCÍA-RODEJA, E. y ILLOBRE, M.L. (1999). Esquemas de razonamiento del alumnado de ESO: Interpretación del posible comportamiento de un sistema cuando intercambia energía con una fuente, en Martínez Losada, C. y García Barros, S. La didáctica de las ciencias. Tendencias actuales. La Coruña: Servicio de Publicacións da Universidade da Coruña.

DOMÍNGUEZ, J.M., GARCÍA-RODEJA, E., PRO BUENO, A. DE y ILLOBRE GONZÁLEZ, M.L. (2001a). Fundamentos para la planificación de una propuesta de enseñanza en la ESO. Cambios en un sistema material. ADAXE. Revista de Estudios e Experiencias Educativas, 17, pp. 311-336.

DOMÍNGUEZ, J.M., GARCÍA-RODEJA, E., PRO BUENO, A. DE y ILLOBRE GONZÁLEZ, M.L. (2001b). Propuesta de enseñanza para el estudio de los cambios en un sistema material, desde la termodinámica y el modelo cinético de partículas. ADAXE. Revista de Estudios e Experiencias Educativas, 17, pp. 337-361

DRIVER, R. y RUSELL, T. (1982). An investigation of the ideas of heat, temperature and change of state of children aged between 8 and 14 years. Londres: University of Leeds and Chelsea College.

DUIT, R. y GLYNN, S. (1996) Mental modelling, en Welford, G., Osborne, J. y Scott, P. (eds.). Research in Science Education in Europe. Londres: The Falmer Press Group.

ERICKSON, G.L. (1989). Calor y temperatura. Revisión de las ideas de los alumnos, en Driver et al. Ideas científicas en la infancia y en la adolescencia. Madrid: MEC-Morata.

ERICKSON, G.L. y TIBERGHIEN, A. (1989). Calor y temperatura. El punto de vista científico, en Driver et al. Ideas científicas en la infancia y en la adolescencia. Madrid: MECMorata.

FURIÓ, C. y HERNÁNDEZ, J. (1983). Ideas sobre los gases en alumnos de 10 a 15 años. Enseñanza de las Ciencias, 1(2), pp. 83-91.

GARCÍA, J.L. y RODRÍGUEZ, C. (1985). Preconcepciones sobre el calor en $2^{\circ}$ de BUP. Enseñanza de las Ciencias, 3(3), pp. 188-193.

GRECA, I.M. y MOREIRA, M.A. (1998). Modelos mentales, modelos conceptuales y modelización. Caderno Catarinense Ensino da Física, 15(2), pp. 107-120.
HIERREZUELO MORENO, J. y MONTERO MORENO, A., (1989). La ciencia de los alumnos. Su utilización en la didáctica de la física y química. Barcelona: Laia-MEC.

HIERREZUELO MORENO, J., MOLINA GONZÁLEZ, E. y MONTERO MORENO, A. (1987). Consideraciones sobre la enseñanza del calor en $2^{\circ}$ de BUP. Enseñanza de las Ciencias, 5(1), pp. 81-83.

LEW, CH.W. y TREAGUST, D.F. (1995). A Predict-ObserveExplain Teaching Sequence for Learning about Students' Understanding of Heat and Expansion Liquids. Australian Science Teachers Journal, 41(1), pp. 68-71.

LLORENS, J.A. (1991). Comenzando a aprender química. Madrid: Visor.

MARÍN, N. y BENARROCH, A. (1994). A comparative study of Piagetian and constructivist work on conceptions in science. International Journal of Science Education, 16(1), pp. 1-15.

MINSKY, M. (1986). La sociedad de la mente: la inteligencia humana a la luz de la inteligencia artificial. Buenos Aires: Galápago.

MITCHELL, A.C. y KELLINGTON, S.H. (1982). Learning difficulties associated with the particulate theory of mater in the Scottish Integrated Science Course. European Journal of Science Education, 4(4), pp. 429-440.

MOREIRA, M.A. (1997). Modelos Mentais. Investigacoes em ensino de Ciencias, 1(3). http://www.if.ufrgs.br/public/ensino/ revista.htm.

NERSSESIAN, N. (1995). Should physicists preach what they practice? Science and Education, 4, pp. 203-226.

NOVICK, S. y NUSSBAUM, J. (1981). Pupils understanding of de particulate nature of matter: a cross-age study. Science Education, 65(2), pp. 187-196.

NUSSBAUM, J. (1989). Classroom conceptual change: philosophical perspectives. Iternational Journal of Science Education, 11 (special issue), pp. 530-540.

PIAGET, J. e INHELDER, B. (1982). El desarrollo de las cantidades en el niño. Barcelona: Hogar del Libro.

POSADA, J.M. (1993). Concepciones de los alumnos de 15-18 años sobre la estructura interna de la materia en el estado sólido. Enseñanza de las Ciencias, 11(1), pp. 12-19.

POSNER, G.J., STRIKE, K.A., HEWSON, P.W. y GERTZOG, W.A. (1982). Accomodation of a Scientific Conception: Toward a Theory of Conceptual Change. Science Education, 62(2), pp. 211-227.

POZO, J.I. (1989). Teorías cognitivas del aprendizaje. Madrid: Morata.

POZO, J.I., GÓMEZ CRESPO, M.A., LIMÓN, M. y SANZ SERRANO, A. (1991). Procesos cognitivos en la comprensión de la ciencia: las ideas de los adolescentes sobre la química. Madrid: Servicio de Publicaciones del MEC.

PRO, A. (1999). Planificación de unidades didácticas por los profesores: análisis de tipos de actividades de enseñanza. Enseñanza de las Ciencias, 17(3), pp. 411-429.

PRO, A., SAURA, O. y SÁNCHEZ, G. (1999). ¿Qué contenidos procedimentales seleccionan los profesores de ciencias cuan- 
do planifican unidades didácticas?, en Martínez y García, $L a$ didáctica de las ciencias experimentales. Tendencias actuales, pp. 115-128. La Coruña: Servicio Publicaciones da Universidadade da Coruña.

PRO, A., SAURA, O. y SÁNCHEZ, G. (2000). ¿Qué actividades de enseñanza utilizan los profesores en formación inicial y los profesores en ejercicio cuando planifican unidades didácticas en ciencias? Investigación en la Escuela, pp. 23-37.

RUMELHART, D.E. (1989). The architecture of mind: a connectionist approach, en Posner, I. (ed.). Fundations of Cognitive Science. Cambridge: The MIT Press.

RUMELHART, D.E. (1997). Hacia una comprensión de la comprensión. Cali: Editorial Universidad del Valle.

RUMELHART, D.E. y ORTONY, A. (1982). La representación del conocimiento en la memoria. Infancia y Aprendizaje, 1920, pp. 115-118.

SCHANCK, R.C. y ABELSON, R.P. (1987). Guiones, planes, metas y entendimiento: un estudio de las estructuras del conocimineto humano. Barcelona: Paidós.

SCHAUBLE, L., KLOPFER, L.E. y RAGHAVAN, K. (1991). Students' transition from an engineering model to a science model of experimentation. Journal of Research in Science Teaching, 18, pp. 859-882.

SHAYER, M. y ADEY, P. (1986). La ciencia de enseñar ciencias. Madrid: Narcea.

SHAYER, M. y WYLAM, H. (1981). The development of the concepts of heat and temperature in 10-13 years olds. Journal of Research in Science Teaching, 8, pp. 419-434.

STAVY, R. (1990). Children's conception of changes in the state of matter: from liquid (or solid) to gas. Journal of Research in Science Teaching, 27(3), pp. 247-266.
STAVY, R. y BERKOWITZ, B. (1980). Cognitive conflicts as a basis for teaching cuantitative aspects of the concept of temperature. Science Education, 64, pp. 679-692.

STRAUSS, S. (1983). Educational-Developmental Psychology and Curriculum Development: The case Heat and Temperature. International Seminar Misconceptions in Science and Mathematics. Ithaca: Cornell University.

SUMMERS, M.K. (1983). Teaching Heat an Analysis of Misconceptions. School Science Review, 64, pp. 670-676.

SUTTON, C. (1996). The scientific model as a form of speech, en Welford, G., Osborne, J. y Scott, P. (eds.). Research in Science Education in Europe. Londres: The Falmer Press Group.

THOMPSON, J.T. (1989). The Sagging Wire. Science Activities, 26 (1), pp. 8-11.

TIBERGHIEN, A. (1980). Un example de restructuration de l'organisation conceptuell à l'occasion d'un enseignement concernant la notion de chaleur, en Compte-rendus de Deuxièmes Journées International sur l'Education Scientifique. Chamonix.

TIBERGHIEN, A. y BARBOUX, M. (1983). Difficulté de la acquisition de le notion de température par les élèves de 6ème, en Compte-rendus des Cinquièmes Journées International sur l'Education Scientifique. Chamonix.

VALCÁRCEL, M.V., PRO, A., BANET, E. y SÁNCHEZ, G. (1990). Problemática didáctica del aprendizaje de las ciencias experimentales. Murcia: Secretariado de Publicaciones de la Universidad de Murcia.

VÁZQUEZ DÍAZ, J. (1987). Algunos aspectos a considerar en la didáctica del calor. Enseñanza de las Ciencias, 5(3), pp. 235-238.

VYGOTSKI, L.S. (1979). El desarrollo de los procesos psicológicos superiores. Barcelona: Crítica.

[Artículo recibido en noviembre de 2001 y aceptado en diciembre de 2002.] 